Stephanie Clarke • Anne Bellmann Thiran •

Philippe Maeder • Michela Adriani • Olivier Vernet •

Luca Regli · Olivier Cuisenaire · Jean-Philippe Thiran

\title{
What and Where in human audition: selective deficits following focal hemispheric lesions
}

Received: 6 February 2002 / Accepted: 25 June 2002 / Published online: 13 September 2002

(C) Springer-Verlag 2002

\begin{abstract}
A sound that we hear in a natural setting allows us to identify the sound source and localize it in space. The two aspects can be disrupted independently as shown in a study of 15 patients with focal right-hemispheric lesions. Four patients were normal in sound recognition but severely impaired in sound localization, whereas three other patients had difficulties in recognizing sounds but localized them well. The lesions involved the inferior parietal and frontal cortices, and the superior temporal gyrus in patients with selective sound localization deficit; and the temporal pole and anterior part of the fusiform, inferior and middle temporal gyri in patients with selective recognition deficit. These results suggest separate cortical processing pathways for auditory recognition and localization.
\end{abstract}

Keywords Sound recognition - Sound localization . Parallel processing - Auditory cortex · Parietal cortex · Temporal cortex

\section{Introduction}

Several lines of evidence suggest that sound recognition and sound localization are processed by neural networks that are distributed in both hemispheres. Activation studies demonstrated that categorization of environmental sounds involves more specifically left prefrontal, tempo-

S. Clarke $(\cdot) \cdot$ A. Bellmann Thiran · M. Adriani

Division de Neuropsychologie, CHUV, 1011 Lausanne,

Switzerland

e-mail: stephanie.clarke@chuv.hospvd.ch

Tel.: +41-21-3141309

Fax: +41-21-3141319

P. Maeder

Service de Radiodiagnostic et Radiologie Interventionnelle,

CHUV, Lausanne, Switzerland

O. Vernet · L. Regli

Service de Neurochirurgie, CHUV, Lausanne, Switzerland

O. Cuisenaire · J.-P. Thiran

Institut de Traitement des Signaux, EPFL, Lausanne, Switzerland ral, parietal and cingulate regions (Engelien et al. 1995), and our current studies have demonstrated that recognition of environmental sounds involves bilaterally regions on the temporal and prefrontal convexities (Maeder et al. 2001). The ability to recognize environmental sounds was found to be deficient following right hemispheric lesions (Spreen et al. 1965; Assal and Aubert 1979; Fujii et al. 1990; Clarke et al. 1996). Combined deficits of recognition of verbal and non-verbal sounds were reported in cases of bilateral (Albert et al. 1972; Motomura et al. 1986; Mendez and Geehan 1988; Buchtel and Stewart 1989) or unilateral left lesions (Pasquier et al. 1991; Clarke et al. 2000). Right hemispheric specialization for sound recognition was suggested by studies on dichotic listening in normal subjects; left ear advantage has been demonstrated for environmental sounds, but also for nonverbal vocalization, emotional intonation and timbre (for review see, e.g., Bradshaw and Nettleton 1981).

In functional studies, sound localization was shown to activate largely distributed cortical networks with an important contribution of the temporal, parietal and prefrontal cortices (Griffiths et al. 1998, 2000; Bushara et al. 1999; Maeder et al. 2001). Some authors suggested a dominance of the right hemisphere (Griffiths and Green 1999; Weeks et al. 1999; Griffiths et al. 2000), whereas others found no evidence for lateralization in auditory spatial processing (Bushara et al. 1999; Woldorff et al. 1999). Focal hemispheric lesions were shown to impair the ability to localize sound sources. Several studies reported mis-localization within the hemispace contralateral to the brain lesion, after right or left hemispheric lesions (Wortis and Pfeffer 1948; Sanchez-Longo and Forster 1958; Klingon and Bontecou 1966; Poirier et al. 1994; Zatorre et al. 1995). Other studies described deficits within the whole field following unilateral lesions (Haeske-Dewick et al. 1996; Zatorre and Penhune 2001) and some advocated either right (Ruff et al. 1981; Bisiach et al. 1984; Tanaka et al. 1999) or left (Pinek et al. 1989) hemispheric specialization for auditory localization. While some authors attributed impaired sound localization exclusively to lesions in temporal lobe (Sanchez- 
Longo and Forster 1958; Efron et al. 1983; Zatorre and Penhune 2001), others questioned its importance in sound localization (Jerger et al. 1972) or reported auditory spatial deficits following parietal lobe lesions (Bisiach et al. 1984; Pinek and Brouchon 1992; Griffiths et al. 1997).

Evidence from recent electrophysiological studies suggests that in non-human primates auditory information relevant to sound recognition and that relevant to localization are processed along two distinct cortical pathways (Rauschecker et al. 1995; Kaas et al. 1999; Romanski et al. 1999; Rauschecker and Tian 2000; Tian et al. 2001). The relevance of these observations to sound processing in man has been questioned (Belin and Zatorre 2000), citing as a major argument the absence of cases with selective deficits.

We present here 15 patients with focal right hemispheric lesion, of whom four had a deficit in sound recognition but not in sound localization and three others a deficit in sound localization but not in sound recognition. This double dissociation clearly supports conclusions drawn from electrophysiological studies in nonhuman primates (Romanski et al. 1999; Rauschecker and Tian 2000; Tian et al. 2001) and from activation studies in humans (Maeder et al. 2001; Warren et al. 2002).

\section{Materials and methods}

\section{Subjects}

Fifteen patients participated in this study (Table 1). All sustained a unilateral right hemispheric lesion 4 months to 4 years prior to the auditory cognitive testing reported here. All had a detailed neuropsychological evaluation as part of their neurorehabilitation program; in all cases only moderate deficits linked to the right hemispheric damage were observed. Two typical case histories are described in detail. Informed consent of the patients and control subjects was obtained according to the Declaration of Helsinki. The study was approved by the Ethics Committee for Clinical Research, University of Lausanne.

JG was a 54-year-old, right-handed man, who suffered a closed head injury characterized by a very short loss of consciousness (1015 s) and no pre- or post-traumatic amnesia. A CT scan was performed 3 days and magnetic resonance imaging (MRI) 3.6 years after the accident; they showed localized damage to the temporal pole and the anterior parts of the fusiform gyrus and the middle and inferior temporal gyri on the right side, without signs of damage or atrophy elsewhere. At the time of testing, 3.4 years after the accident, neuropsychological assessment revealed moderate memory and executive impairments, mild word finding difficulties, constructional apraxia and difficulties in orientation on geographical maps. A moderate impairment in face recognition, present at the initial stage, had completely regressed.

E.S. was a 64-year-old right-handed woman who suffered subarachnoidal hemorrhage from a ruptured right middle cerebral artery aneurysm. The aneurysm was successfully clipped, but within the first postoperative week the patient suffered arterial spasms of the middle cerebral artery which caused lesions of the anterior and posterior parts of the supratemporal plane, the insula, the middle portion of the inferior frontal gyrus, and the supramarginal and angular gyri. At the time of testing, 2 months after the ischemic insult, the patient had constructional apraxia and residual executive impairments. Mild manifestations of visual hemineglect and deficits in visuospatial memory, present at the initial stage of illness, had regressed completely.

Recognition of environmental sounds

The test consisted of 50 samples of environmental sounds each of which lasted $7 \mathrm{~s}$ and was accompanied by a multiple-choice display of five drawings: the target; an object acoustically and semantically related to the sound; semantically related; acoustically related; and an object neither acoustically nor semantically related. The subject had to indicate the correct sound source. A detailed description of the test and normative data on 60 control subjects have been published previously (Clarke et al. 1996). The average number of correct replies among the normal subjects was $46.88(\mathrm{SD}=2.45)$. The limit of normal performance was set 2 SD below the mean, which corresponded to a score of 42 .
Table 1 Performance in sound recognition and sound localization in a series of 15 consecutive patients with focal right hemispheric lesions, including ES and JG. All patients except MIP were right handed. Localization measures are the relative score, expressed as $z$-score, and the number of alloacuses. Alloacuses were never found in the control population $(N=60)$. Deficient results are in bold $(B G$ basal ganglia, $C C$ corpus callosum, $C H I$ closed head injury, $F$ frontal, Ins insula, $O$ occipital, $P$ parietal, $T$ temporal, Tha thalamus)

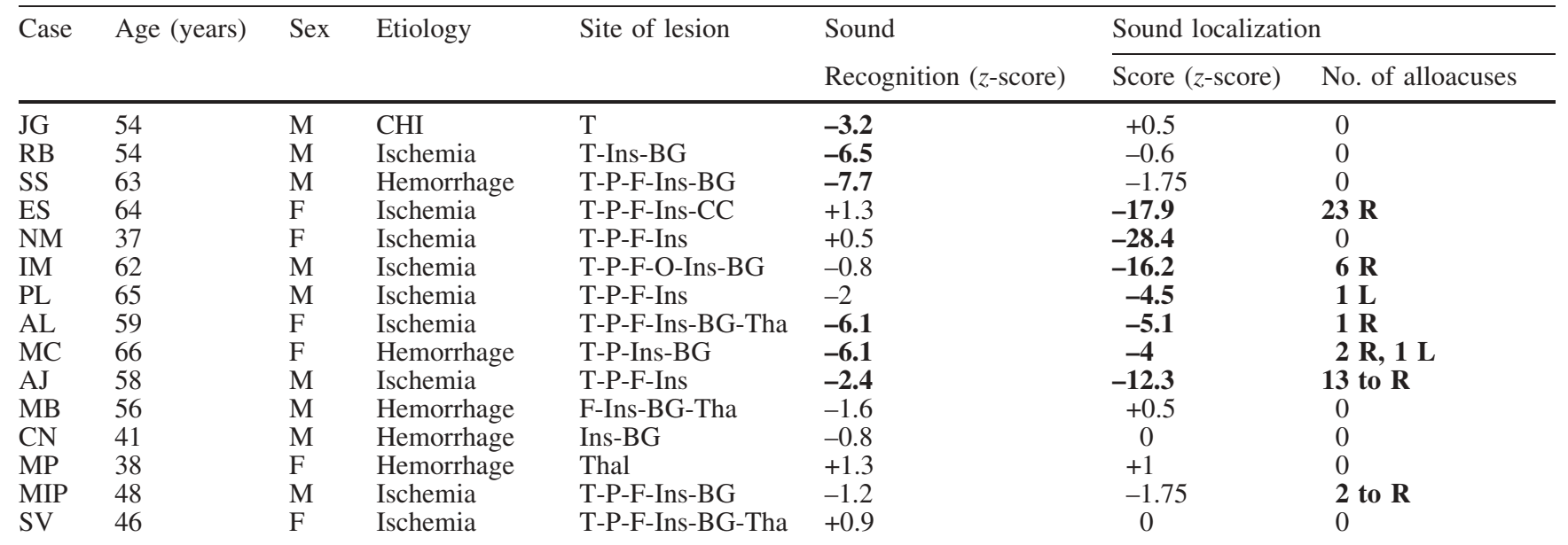


A detailed description of this test and normative data on 60 control subjects have been reported elsewhere (Clarke et al. 2000; Bellmann et al. 2001). Sound lateralization was simulated with differences in interaural time. The stimulus was a 2 -s broadband bumblebee sound, shaped with $100 \mathrm{~ms}$ rising and falling times, and presented through earphones. One central and four lateral positions, two in each hemispace, were simulated. The lateral positions were created by delaying the left or right channel by $0.3 \mathrm{~ms}$ or $1 \mathrm{~ms}$. Sixty items, 12 in each position, were presented in pseudorandom order. The patients were asked to indicate the perceived position on their head with their ipsilesional hand (same procedure as Altman et al. 1979; Bisiach et al. 1984). A graduated half-circle fixed on the headphones was used to determine the angular value of the position (from $0^{\circ}$ at the vertex, to $90^{\circ}$ at each ear). As a measure of overall performance (max. 59), the relative positions attributed to two consecutive stimuli were compared; a response was counted as correct when a stimulus was correctly placed to the left or the right of the previous stimulus in correspondence with the difference in interaural temporal discrepancy or within $\pm 10^{\circ}$ of the previous location for identical interaural temporal values. Alloacuses (perception of stimuli as shifted to the other side of the mediosagittal plane) were also recorded. The patients' individual scores were converted into $z$-scores relative to the mean and standard deviation of the control population $($ mean $=57.15, \mathrm{SD}=$ 1.79); the limit of normal performance was set 2 SD below the mean $(z$-score $<-2)$.

\section{Anatomical evaluation}

The normalized coordinate system of Talairach and Tournoux (1988) was adopted for the comparison of sites of lesions with anatomical data from normal postmortem material. Lesions were delineated on MRI for patients J.G. and E.S., and were displayed and analyzed using OrthoViewer, an image visualization and processing software program, developed in our laboratory, able to display orthogonal views of a 3D image. The OrthoViewer is implemented to superimpose the Talairach grid on the images based on a manual selection of the anterior and posterior commissures and of the anterior, posterior, top, bottom and lateral limits of the brain

\section{Results}

\section{Sound recognition}

Patient JG identified correctly 39 out of 50 items; his performance was deficient, 3.20 SD below the mean of normal subjects (Fig. 1a). His errors consisted in choosing items that were both semantically and acoustically linked to the target (eight errors), items that were semantically but not acoustically linked to the target (one error), or items that were acoustically but not semantically linked to the target (two errors); no items were chosen that were neither acoustically nor semantically linked to the target. Errors were equally distributed among the semantic categories represented (animals, common objects, musical instruments, vehicles, tools, human and natural sounds). Among the errors, three represented items never selected by the controls, and another one was an error made by only one of the 60 normal subjects.

The other patient, ES, identified correctly all 50 items, which corresponds to an excellent performance, 1.25 SD above the mean (Fig. 1a).
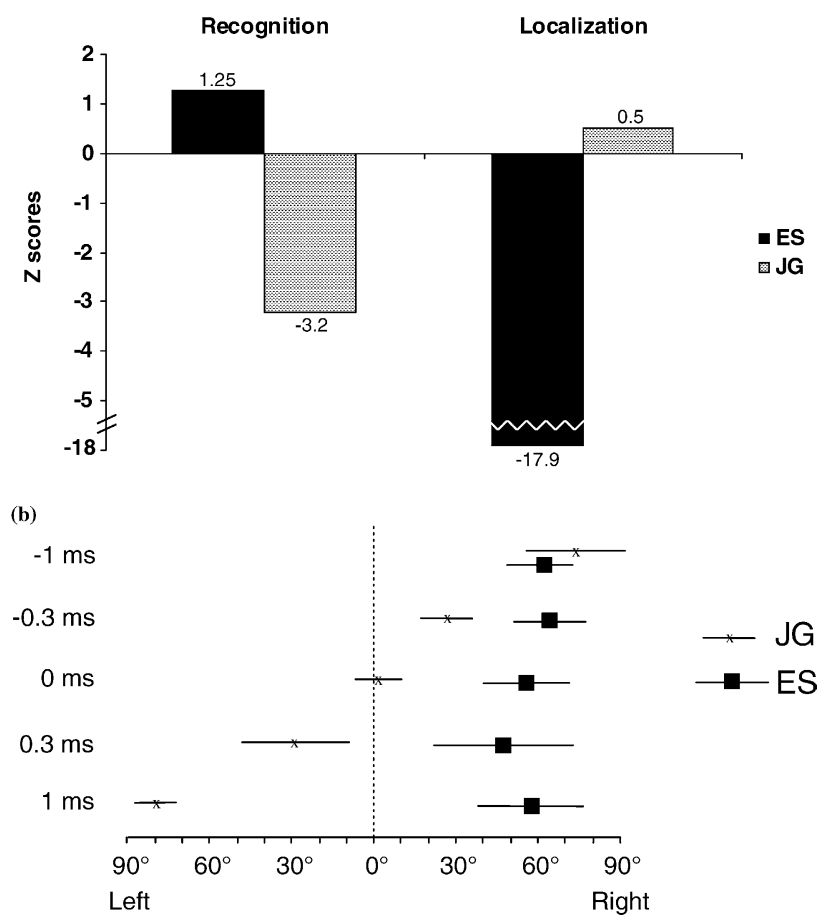

Fig. 1a, b Sound recognition and sound localization following focal brain damage. a Performance of patients ES and JG as compared to normal population by means of $z$-scores; -2 denotes the limit of normal performance. Note that JG was deficient and ES normal in sound recognition, whereas the reverse was the case for sound localization. b Performance in sound localization. Interaural time differences (left minus right ear) are indicated on the $y$-axis, angular values of the indicated positions on the $x$-axis. Mean value and SD of 12 presentations are indicated for each interaural temporal difference

\section{Sound localization}

Patient JG performed extremely well (Fig. 1). When compared to the normal population his performance was in the upper range, with an overall performance score of $58(z$-score $=0.5)$.

Patient ES was severely deficient on sound localization. She reported being unable to perceive different sound positions and localized all stimuli within the same part of the right hemifield (Fig. 1b). She was also unable to discriminate different positions; she reported that all positions sounded the same to her. Left hemifield stimuli were perceived on the right side (23 alloacuses), which was never observed in normal subjects. Judgement of the relative positions of two consecutive stimuli was correct at a random level (25/59), which led to a score 17.9 SD below the mean of the control population (Fig. 1a). 
Fig. 2 Top Lesions in JG and $\mathrm{ES}$ as analyzed in Talairach space. JG had a right anterior temporal lesion that involved the temporal pole and the anterior parts of middle and inferior temporal and fusiform gyri; the supratemporal plane was entirely spared. Patient ES had a right temporo-fronto-parietal lesion that involved the inferior parietal lobule, middle portion of the inferior frontal gyrus, Heschl's gyrus, and parts of planum temporale and polare, but spared the temporal convexity. Bottom Three-dimensional views of JG's brain with the lesion in green (left), associated with selective deficit of sound recognition, and of ES's brain with the lesion in red (right), associated with selective deficit in localization

\section{Horizontal Sagittal Coronal}
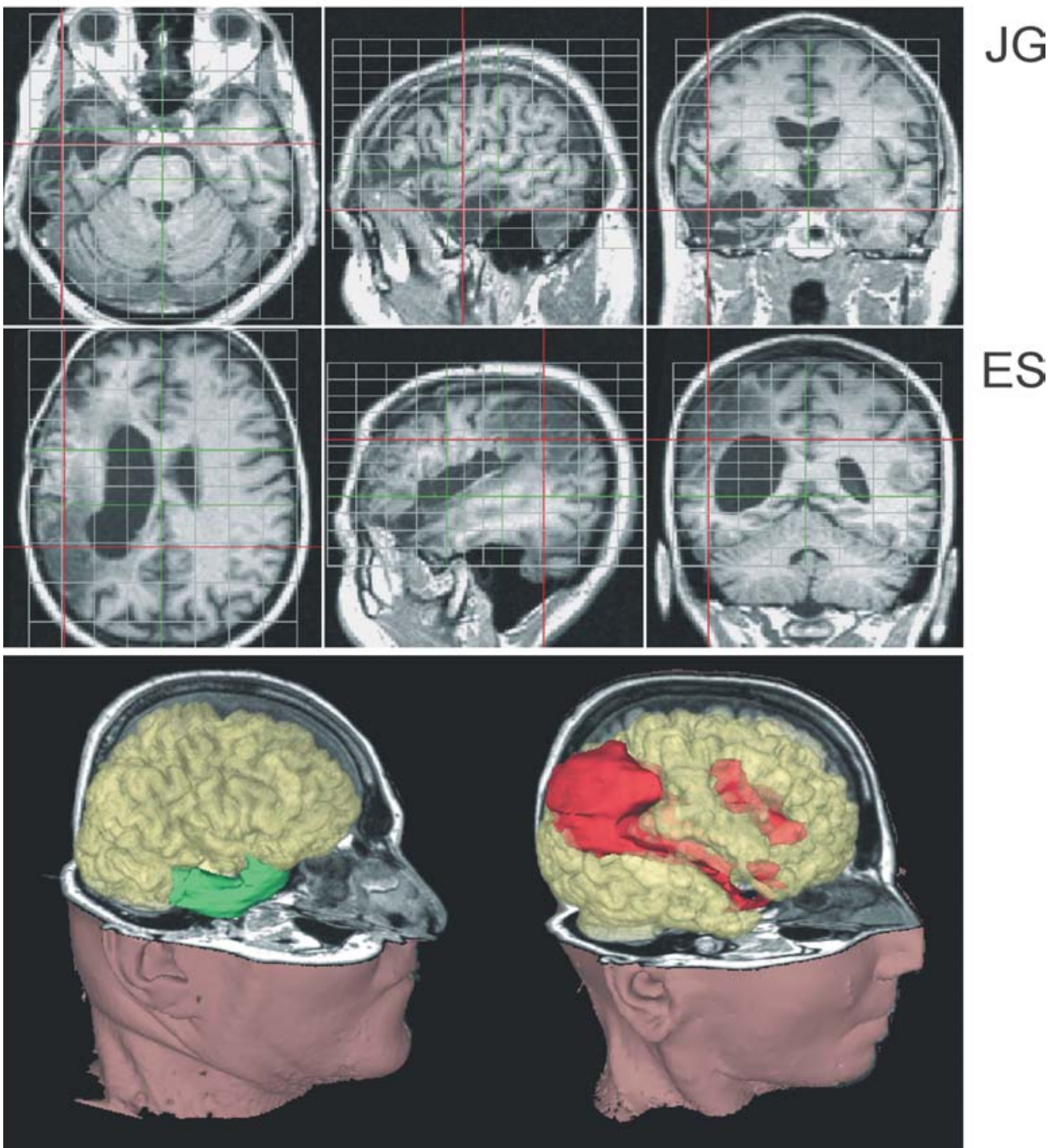

Lesions associated with selective deficit in sound recognition or sound localization

Patient JG had a right anterior temporal lesion that involved the temporal pole and the anterior parts of middle and inferior temporal and fusiform gyri; the supratemporal plane was entirely spared. Patient ES had a right temporo-fronto-parietal lesion that involved the posterior part of the inferior parietal lobule, middle portion of the inferior frontal gyrus, Heschl's gyrus, parts of the plana temporale and polare, but spared the temporal convexity (Fig. 2).
Selective deficits in sound recognition or sound localization occur frequently following right hemispheric lesions

Evaluation of a series of 15 consecutive patients with focal right hemispheric lesions (including JG and ES; Table 1) revealed that two more patients had a deficit in sound recognition but not sound localization (RB, SS) and three more patients had a deficit in sound localization but not sound recognition (NM, IM, PL). Three patients had a combined deficit in sound recognition and sound localization $(\mathrm{AL}, \mathrm{MC}, \mathrm{AJ})$ and five patients were normal in both (MB, CN MP, MIP, SV).

Lesions that were associated with selective deficit in sound recognition were centered on the anterior part of the temporal lobe, while lesions associated with selective deficit in sound localization were centred on the parietofrontal convexity (Fig. 3). 
Fig. 3 Superimposed lesions of the four right-damaged patients with selective deficit for localization (left column) and of the three right-damaged patients with selective deficit for recognition (right column). The lesions are represented in

Talairach space, sections c and d. Gray levels indicate the number of patients in whom a given Talairach cube was completely or partially damaged
Selective deficit in auditory localization $(\mathrm{N}=4)$
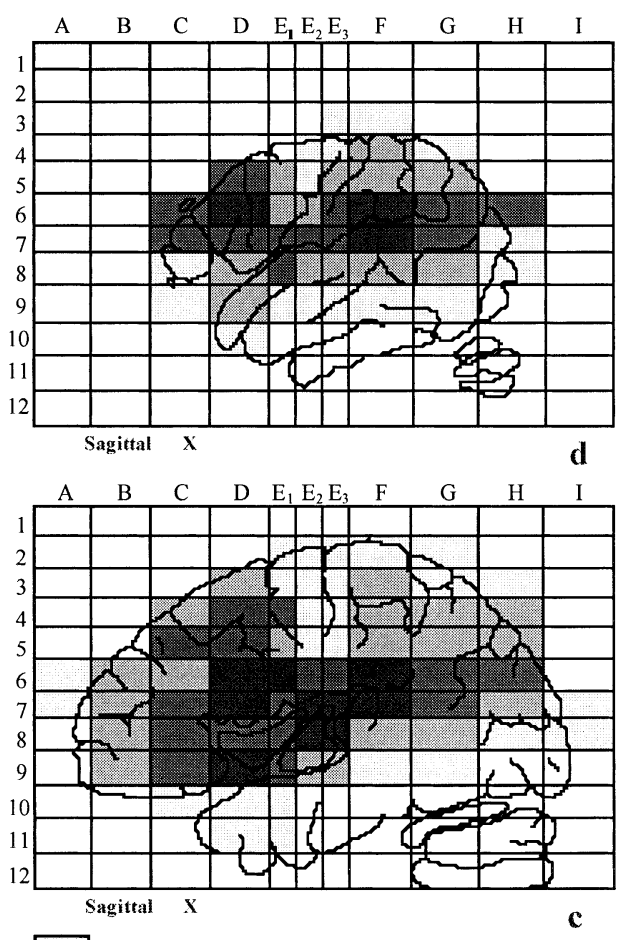

Selective deficit in auditory recognition $(\mathrm{N}=3)$
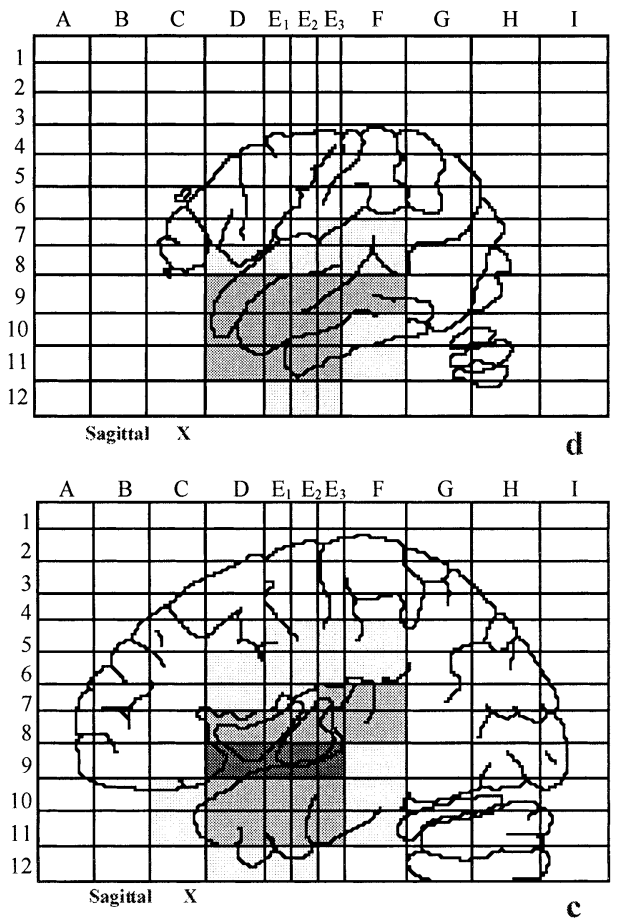

\section{Discussion}

A sound that we hear in a natural setting allows us to identify the sound source and to localize it simultaneously in space. Evidence from electrophysiological studies in non-human primates suggests that the two aspects are processed within distinct cortical areas. Neurons in areas lateral to the primary auditory area were shown to be selective for stimuli with specific frequency and intensity modulations, corresponding most likely to invariants that allow identification of sound sources (Rauschecker et al. 1995). Neurons in areas posterior to the primary auditory cortex appeared to be involved in sound localization (Leinonen et al. 1980; Rauschecker et al. 1997; Recanzone et al. 2000) and those in areas anterior to it in sound identification (Tian et al. 2001).

The dichotomy between auditory What and Where pathways, as described in non-human studies (Rauschecker 1998; Romanski et al. 1999; Kaas et al. 1999; Rauschecker and Tian 2000; Tian et al. 2001), is subject to discussion in human studies (Belin and Zatorre 2000). Recent functional magnetic resonance imaging (fMRI) (Maeder et al. 2001) and electrophysiological studies (Alain et al. 2001; Anourova et al. 2001) in humans demonstrated distinct cortical networks involved in spatial and non-spatial auditory processing. While the existence of a ventral pathway dedicated to sound recognition - the What pathway - is generally accepted, the specialization of the dorsal one remains more controversial and it has been proposed that the dorsal pathway plays a role in the analysis of spectral motion rather than sound localization (Belin and Zatorre 2000). A recent study has, however, demonstrated a role of the caudal belt in the processing of auditory motion in space (Warren et al. 2002).

In man, previously reported cases are compatible with a relative independence in the processing of sound recognition and sound localization. There are three reports of auditory agnosia without auditory localization deficits (Spreen et al. 1965; Jerger et al. 1972; Fujii et al. 1990) following right or bilateral lesions. Selective impairment in auditory motion perception was reported in one case following a right hemispheric lesion that included the insula and parietal convexity (Griffiths et al. 1996, 1997). A relative independence of sound recognition and sound localization is also suggested by a study in normal subjects, which showed that short-term memory for content or location was affected differentially by specific auditory interference tasks (Clarke et al. 1998). More recently an activation study revealed separate cortical networks for sound recognition and sound localization; sound recognition activated selectively regions on the left and right temporal and on left prefrontal convexities and sound localization bilaterally regions on parietal and frontal convexities (Maeder et al. 2001).

The present study demonstrates that damage to either of the specialized networks disrupts selectively the corresponding ability. Patient JG had difficulties in 
recognizing sounds but localized them extremely well. His impairment in auditory recognition was not part of a general semantic disorder: he could easily recognize and name the pictures, even those belonging to sounds he misidentified. Moreover, within the auditory modality, the errors were not only of semantic, but also acoustical type. More detailed auditory testing (not presented here) showed normal performance in non-verbal auditory asemantic recognition (the ability to match two acoustically different samples of the same object). His deficit appeared to be restricted to the ability to extract meaning from auditory non-verbal stimuli. Patient ES was excellent in sound recognition but had great difficulties in localization. Her selective impairment in auditory spatial orientation was not part of a more general spatial disorder, since her visual spatial functions were within normal limits. The functional double dissociation between auditory localization and recognition was coupled with a dissociation in the cerebral regions responsible for the observed deficits. The recognition deficit was associated with damage to the temporal pole and convexity (middle and inferior), which supports Scott and colleagues' proposal of a ventral pathway for the recognition of intelligible speech (Scott et al. 2000). The localization deficit was associated with a dorsal temporo-parietofrontal lesion. Given the extent of the lesion, it is not possible to determine precisely which parts of it were responsible for the deficit. However, activation data emphasize the involvement of an extensive cortical network dedicated to auditory spatial processing (Griffiths et al. 2000), and the lesions of our other patients with selective localization impairment also involved temporal as well as more dorsal parietal and frontal regions. The association of temporal, parietal and frontal lesions might be necessary to result in a deficit of auditory localization.

Seven cases of this study demonstrate a double dissociation between sound recognition and localization, and emphasize the differential role of the temporal convexity and parieto-prefrontal convexity in audition (Fig. 3). Recently we have also reported a similar double dissociation with similar anatomical substrate following relatively large left hemispheric lesions (Clarke et al. 2000). Two patients with lesions centred on the temporal convexity were deficient in sound recognition and normal in sound localization, while one patient with a large lesion including the parietal and frontal convexities was deficient in sound localization and normal in sound recognition. These data suggest that auditory non-verbal information is processed in each hemisphere along two parallel cortical pathways, a ventral one dedicated to recognition, and a dorsal one dedicated to the spatial processing of sounds.

The existence of What and Where pathways in both hemispheres raises the question of a putative hemispheric specialization in sound recognition or sound localization. In an earlier study we have shown sound recognition deficits following right or left unilateral lesions (Clarke et al. 1996); a higher percentage of sound identification deficits was observed after right than left lesions, while deficits concerning sound stimulus segregation were more frequently associated with left than right lesions. Sound localization depends on processing in either hemisphere, although right hemispheric lesions appear to yield greater deficits, which can involve the whole space, as shown in the present paper, while left hemispheric lesions were found to involve mainly the right hemispace (Clarke et al. 2000).

The existence of separate processing pathways is supported by anatomical studies of the human auditory cortex. The supratemporal plane is likely to comprise several distinct areas, as indicated by changes in cortical architecture (Galaburda and Sanides 1980), and area-like structures were identified with cytochrome oxidase and acetylcholinesterase histochemistry (Rivier and Clarke 1997). Recent connectivity (Tardif and Clarke 2001) and activation studies (Hashimoto et al. 2000; Wessinger et al. 2001) suggest hierarchical organization of these areas within the ventral, recognition pathway. Furthermore, human primary auditory cortex was shown to contain distinct intra-areal compartments, cytochrome oxidase dark and light stripes, that are reminiscent of compartments within human V1 and V2 and that may represent an early segregation of different processing pathways (Clarke and Rivier 1998).

In conclusion, our results provide evidence that impairments in auditory localization and auditory recognition can occur separately, and depend on different cortical lesion sites. We propose the existence, in the auditory modality, of a 'what' ventral pathway, going anteriorly and laterally down the temporal convexity, and of a 'where' dorsal pathway involving a temporo-parietofrontal network (Fig. 2, bottom).

Acknowledgements We thank Prof. F. de Ribaupierre for many helpful discussions on stimulus production; Miss S. Pavin for assistance in testing normal subjects, and Drs. Claire Bindschaedler and Rolf Frischknecht for data on neuropsychological and general rehabilitation of patients. This work was supported by the Swiss National Science Foundation grants 3100-064085.00 and 313851173.97, a Biomed grant from UNIL-EPFL-UNIGE, and a Théodore-Ott grant.

\section{References}

Alain C, Arnott SR, Hevenor S, Graham S, Grady CL (2001) "What" and "where" in the human auditory system. Proc Natl Acad Sci U S A 98:12301-12306

Albert ML, Sparks R, Von Stockert T, Sax D (1972) A case study of auditory agnosia: linguistic and non-linguistic processing. Cortex 8:427-443

Altman J, Balonov L, Delgin V (1979) Effects of unilateral disorder of the brain hemispheric function in man on directional hearing. Neuropsychologia 17:295-301

Anourova I, Nikouline VV, Ilmoniemi RJ, Hotta J, Aronen HJ, Carlson S (2001) Evidence for dissociation of spatial and nonspatial auditory information processing. Neuroimage 14:1268-1277

Assal G, Aubert C (1979) La reconnaissance des onomatopées et des cris d'animaux lors de lésions focalisées du cortex cérébral. Rev Neurol 135:65-73 
Belin P, Zatorre RJ (2000) 'What', 'where' and 'how' in auditory cortex. Nat Neurosci 3:965-966

Bellmann A, Meuli R, Clarke S (2001) Two types of auditory neglect. Brain 124:676-687

Bisiach E, Cornacchia L, Sterzi R, Vallar G (1984) Disorders of perceived auditory lateralization after lesions of the right hemisphere. Brain 107:37-52

Bradshaw JL, Nettleton NC (1981) The nature of hemispheric specialization in man. Behav Brain Sci 4:51-91

Buchtel HA, Stewart JD (1989) Auditory agnosia: aperceptive or associative disorder? Brain Lang 37:12-25

Bushara KO, Weeks RA, Ishii K, Catalan M-J, Tian B, Rauschecker JP, Hallett M (1999) Modality-specific frontal and parietal areas for auditory and visual spatial localization in humans. Nat Neurosci 2:759-766

Clarke S, Rivier F (1998) Compartments within human primary auditory cortex: evidence from cytochrome oxidase and acetylcholinesterase staining. Eur J Neurosci 10:741-745

Clarke S, Bellmann A, de Ribaupierre F, Assal G (1996) Nonverbal auditory recognition in normal subjects and braindamaged patients: evidence for parallel processing. Neuropsychologia 34:587-603

Clarke S, Adriani M, Bellmann A (1998) Distinct short-term memory systems for sound content and sound localization. Neuroreport 9:3433-3437

Clarke S, Bellmann A, Meuli RA, Assal G, Steck AJ (2000) Auditory agnosia and auditory spatial deficits following left hemispheric lesions: evidence for distinct processing pathways. Neuropsychologia 38:797-807

Efron R, Crandall PH, Koss B, Divenyi PIL, Yund EW (1983) Central auditory processing. III. The 'cocktail party' effect and anterior temporal lobectomy. Brain Lang 19:254-263

Engelien A, Silbersweig D, Stern E, Huber W, Döring W, Frith C, Frackowiak RS (1995) The functional anatomy of recovery from auditory agnosia. A PET study of sound categorization in a neurological patient and normal controls. Brain 118:13951409

Fujii T, Fukatsu R, Watabe S, Ohnuma A, Teramura K, Saso S, Kogure K (1990) Auditory sound agnosia without aphasia following a right temporal lobe lesion. Cortex 26:263-268

Galaburda A, Sanides F (1980) Cytoarchitectonic organization of the human auditory cortex. J Comp Neurol 190:597-610

Griffiths TD, Green GGR (1999) Cortical activation during perception of a rotating wide-field acoustic stimulus. Neuroimage 10:84-90

Griffiths TD, Rees A, Witton C, Shakir RA, Henning GB, Green GGR (1996) Evidence for a sound movement area in the human cerebral cortex. Nature 383:425-427

Griffiths TD, Rees A, Witton C, Cross PM, Shakir RA, Green GGR (1997) Spatial and temporal auditory processing deficits following right hemisphere infarction: a psychophysical study. Brain 120:785-794

Griffiths TD, Büchel C, Frackowiak RSJ, Patterson RD (1998) Analysis of temporal structure in sound by the human brain. Nat Neurosci 1:422-427

Griffiths TD, Green GGR, Rees A, Rees G (2000) Human brain areas involved in the analysis of auditory movement. Hum Brain Mapp 9:72-80

Haeske-Dewick H, Canavan GN, Hömberg V (1996) Sound localization in egocentric space following hemispheric lesions. Neuropsychologia 34:937-942

Hashimoto R, Homae F, Nakajima K, Miyashita Y, Sakai KL (2000) Functional differentiation in the human auditory and language areas revealed by a dichotic listening task. Neuroimage 12:147-158

Jerger J, Lovering L, Wertz M (1972) Auditory disorder following bilateral temporal lobe insult: report of a case. J Speech Hear Disord 37:523-535

Kaas JH, Hackett TA, Tramo MJ (1999) Auditory processing in primate cerebral cortex. Curr Opin Neurobiol 9:164-170

Klingon GH, Bontecou DC (1966) Localization in auditory space. Neurology 16:879-886
Leinonen L, Hyvarinen J, Sovijarvi ARA (1980) Functional properties of neurons in the temporo-parietal association cortex of awake monkey. Exp Brain Res 39:203-215

Maeder P, Meuli R, Adriani M, Bellmann A, Fornari E, Thiran J-P, Pittet A, Clarke S (2001) Distinct pathways involved in sound recognition and localization: a human fMRI study. Neuroimage 14:802-816

Mendez MF, Geehan GR (1988) Cortical auditory disorders: clinical and psychoacoustic features. J Neurol Neurosurg Psychiatry 51:1-9

Motomura N, Yamadori A, Mori E, Tamaru F (1986) Auditory agnosia: analysis of a case with bilateral subcortical lesions. Brain 109:379-391

Pasquier F, Leys D, Steinling M, Guieu JD, Petit H, Cambier J (1991) Agnosie auditive unilatérale droite consécutive à une hémoragie lenticulaire gauche. Rev Neurol 147:129-137

Pinek B, Brouchon M (1992) Head turning versus manual pointing to auditory targets in normal subjects and in subjects with right parietal damage. Brain Cogn 18:1-11

Pinek B, Duhamel JR, Cavé Ch, Brouchon M (1989) Audio-spatial deficits in human: differential effects associated with left versus right hemisphere parietal damage. Cortex 25:175-186

Poirier P, Lassonde M, Villemure JG, Geoffroy G, Lepore F (1994) Sound localization in hemispherectomized patients. Neuropsychologia 32:542-553

Rauschecker JP (1998) Cortical processing of complex sounds. Curr Opin Neurobiol 8:516-521

Rauschecker JP, Tian B (2000) Mechanisms and streams for processing of "what" and "where" in auditory cortex. Proc Natl Acad Sci U S A 97:11800-11806

Rauschecker JP, Tian B, Hauser M (1995) Processing of complex sounds in the macaque nonprimary auditory cortex. Science 268:111-114

Rauschecker JP, Tian B, Pons T, Mishkin M (1997) Serial and parallel processing in rhesus monkey auditory cortex. J Comp Neurol 382:89-103

Recanzone GH, Guard DC, Phan ML, Su TK (2000) Correlation between the activity of single auditory cortical neurons and sound-localization behavior in the macaque monkey. J Neurophysiol 83:2723-2739

Rivier F, Clarke S (1997) Cytochrome oxidase, acetylcholinesterase and NADPH-diaphorase staining in human supratemporal and insular cortex: evidence for multiple auditory areas. Neuroimage 6:288-304

Romanski LM, Tian B, Fritz J, Mishkin M, Goldman-Rakic PS, Rauschecker JP (1999) Dual streams of auditory afferents target multiple domains in the prefrontal cortex. Nat Neurosci 2:1131-1136

Ruff RM, Hersch NA, Pribram KH (1981) Auditory spatial deficits in the personal and extra-personal frames of reference due to cortical lesions. Neuropsychologia 19:435-443

Sanchez-Longo LP, Forster FM (1958) Clinical significance of impairment of sound localization. Neurology 8:119-125

Scott SK, Blank CC, Rosen S, Wise RJS (2000) Identification of a pathway for intelligible speech in the left temporal lobe. Brain 123:2400-2406

Spreen O, Benton AL, Fincham RW (1965) Auditory agnosia without aphasia. Arch Neurol 13:84-92

Talairach J, Tournoux P (1988) Co-planar stereotaxic atlas of the human brain. Georg Thieme, Stuttgart

Tanaka H, Hachisuka K, Ogata H (1999) Sound lateralisation in patients with left or right cerebral hemispheric lesions: relation with unilateral visuospatial neglect. J Neurol Neurosurg Psychiatry 67:481-486

Tardif E, Clarke S (2001) Intrinsic connectivity of human auditory areas: a tracing study with DiI. Eur J Neurosci 13:1045-1050

Tian B, Reser D, Durham A, Kustov A, Rauschecker JP (2001) Functional specialization of rhesus monkey auditory cortex. Science 292:290-293

Warren JD, Zielinski BA, Green GGR, Rauschecker JP, Griffiths TD (2002) Perception of sound-source motion by the human brain. Neuron 34:139-148 
Weeks RA, Aziz-Sultan A, Bushara KO, Tian B, Wessinger CM, Dang N, Rauschecker JP, Hallett M (1999) A PET study of human auditory spatial processing. Neurosci Lett 262:155-158

Wessinger CM, VanMeter J, Tian B, Van Lare J, Pekar J, Rauschecker JP (2001) Hierarchical organization of the human auditory cortex revealed by functional magnetic resonance imaging. J Cogn Neurosci 13:1-7

Woldorff MG, Tempelmann C, Fell J, Tegeler C, GaschlerMarkefski B, Hinrichs H, Heinz HJ, Scheich H (1999) Lateralized auditory spatial perception and the contralaterality of cortical processing as studied with functional magnetic resonance imaging and magnetoencephalography. Hum Brain Mapp 7:49-66

Wortis SB, Pfeffer AZ (1948) Unilateral auditory-spatial agnosia. J Nerv Ment Dis 108:181-186

Zatorre RJ, Penhune VB (2001) Spatial localization after excision of human auditory cortex. J Neurosci 21:6321-6328

Zatorre RJ, Ptito A, Villemure JG (1995) Preserved auditory spatial localization following cerebral hemispherectomy. Brain 118:879-889 